\title{
Mid-Infrared Spectroscopic Investigation of the Perfect Vitrification of Poly(ethylene glycol) Aqueous Solutions
}

\author{
Makoto Gemmei-Ide, ${ }^{*}$ Takashi Miyashita, Shigehiro Kagaya, and Hiromi Kitano* \\ Department of Environmental Applied Chemistry, Graduate School of Science and Engineering, \\ University of Toyama, 3190 Gofuku, Toyama 930-8555, Japan
}

*To whom correspondence should be addressed

(M.G.-I.): E-mail: ide@eng.u-toyama.ac.jp; Tel: +81-76-445-6870; Fax: +81-76-445-6703

(H.K.): E-mail: kitano@eng.u-toyama.ac.jp; Tel: +81-76-445-6868; Fax: +81-76-445-6703

\begin{abstract}
Crystallization/recrystallization behaviors of poly(ethylene glycol) (PEG) aqueous solutions with water contents ( $W_{\mathrm{C}}$ 's) of $\sim 36-51 \mathrm{wt} \%$ were investigated by temperature-variable mid-infrared spectroscopy. At a $W_{\mathrm{C}}$ of $43.2 \mathrm{wt} \%$, crystallization/recrystallization of water and PEG were not observed. At this specific $W_{\mathrm{C}}$ value $\left(W_{\mathrm{CPV}}\right)$, perfect vitrification occurred. Below and above the $W_{\mathrm{CPV}}$ value, crystallization/recrystallization behaviors changed drastically. The crystallization temperature below $W_{\mathrm{CPV}}(237 \mathrm{~K})$ was $\sim 10 \mathrm{~K}$ greater than that above $W_{\mathrm{CPV}}(226 \mathrm{~K})$. Recrystallization above and below $W_{\mathrm{CPV}}$ occurred in one $(213 \mathrm{~K})$ and two $(198$ and $210 \mathrm{~K})$ steps, respectively. These findings resulted from the difference in the (re)crystallization behaviors of water molecules associated with PEG chains with helical and random-coil conformations. These two types of water molecules might have limiting concentrations for their (re)crystallization, indicating that perfect vitrification might have occurred when the concentrations of the two types of water molecules were less than the limiting concentrations of their (re)crystallization.
\end{abstract}

\section{Introduction}

Poly(ethylene glycol) (PEG) $\left(-\left(\mathrm{OCH}_{2} \mathrm{CH}_{2}\right)_{n}{ }^{-}\right)$, also called poly(oxyethylene) (POE) or poly(ethylene oxide) (PEO), is a synthetic polymer with various unique properties and is one of the most extensively studied polymers. PEG is used not only for industrial, medical and cosmetic applications, such as lubricants in metal processing, matrices for medical reagents, and emulsifying agents, respectively, but also as a biocompatible material ${ }^{1-6}$ that strongly suppresses nonspecific protein adsorption and cell adhesion. The formation of a surface diffusion layer based on hydrated PEG chains has been proposed as the origin of the polymer's biocompatibility. ${ }^{711}$ The hydration structure of PEG chains has been of interest because of the extremely high water solubility of PEG; PEG is freely soluble in water, whereas PEG analogues such as poly (oxymethylene) $\left(-\left(\mathrm{OCH}_{2}\right)_{n}-\right)$ and poly(propylene glycol) $\left(-\left(\mathrm{OCH}_{2} \mathrm{CH}\left(\mathrm{CH}_{3}\right)\right)_{n}-\right)$ are insoluble in water. ${ }^{12}$ Many investigations to reveal the hydration structure of the PEG-water binary system have been carried out using various experimental and computational methods including viscosity measurements, ${ }^{13-15}$ quasielastic neutron scattering, ${ }^{16,17}$ nuclear magnetic resonance, ${ }^{18}$ Raman $^{19-21}$ and infrare ${ }^{22-24}$ spectroscopies, and molecular-dynamics ${ }^{25-32}$ and density-functional-theory ${ }^{33}$ simulations. In many of the cases cited here, the hydration structure has been discussed at and/or above $298 \mathrm{~K}$, that is, in the liquid state.

Differential scanning calorimetry (DSC), ${ }^{34-41}$ on the other hand, has also been frequently used to investigate interactions between PEG and water molecules. Differing from the aforementioned methods, DSC analysis cannot be used to investigate the state of water and PEG in the liquid state but has revealed some unique thermal properties of the PEG-water binary system. It has provided evidence for a typical eutectic system, for the crystallization of water during heating, that is, recrystallization of water over a wide range of water content $\left(W_{\mathrm{C}}\right)$ values of $10-95 \mathrm{wt} \%$, and for perfect vitrification at a specific $W_{\mathrm{C}}$ value. These phenomena depend strongly on the molecular weight of PEG and were observed for PEG molecules with molecular weights from 1000-2000. Over the course of nearly a decade, this recrystallization phenomenon (recrystallization of water) has received extensive attention in the field of biomaterials, although it has also been observed for various aqueous polymer solutions such as poly( $N$-vinyl pyrrolidone $){ }^{42}$ ten different types of polysaccharides, ${ }^{43-45}$ and non-denatured proteins (bovine serum albumin ${ }^{46}$ and cytochrome $\mathrm{c}^{47}$ ). This attention was first triggered by a series of reports on the thermal properties of water sorbed on poly(2-methoxyethyl acrylate) (PMEA), which is a non-water-soluble polymer; recrystallization of water was observed for hydrated PMEA, which possesses a highly effective blood-compatible property (biocompatibility). ${ }^{48-50}$ The research group that reported this finding hypothesized that polymers demonstrating recrystallizability of water are biocompatible; conversely, recrystallizable water makes materials biocompatible. The fact that PMEA and PEG possess recrystallizable water and high biocompatibility has often been referenced to support this hypothesis, implying that the hydration structures of PMEA and PEG are the same. Confirmation of the hypothesis is unclear, but it is difficult to predicate it, because 
recrystallizable water is also found with non-biocompatible polymers such as poly(methyl acrylate), poly(n-butyl methacrylate), poly(ethoxyethyl acrylate), poly(ethoxyethyl methacrylate), and polystyrene. ${ }^{51-53}$

A decisive difference, furthermore, between the PEG-water and PMEA-water systems has been reported. Hung and Nishinari demonstrated that an aqueous solution of PEG with a molecular weight of 1540 had a specific $W_{\mathrm{C}}$ at which no crystallization and no recrystallization occurred, that is, at which perfect vitrification occurred. Figure 1 shows the recrystallization and fusion enthalpies of a PEG-water system with various $W_{\mathrm{C}}$ values measured via DSC. At a $W_{\mathrm{C}}$ of $\sim 40 \mathrm{wt} \%$, no recrystallization or fusion of PEG, no eutectic mixture (PEG-water), and no water were observed; it demonstrated perfect vitrification. In usual polymer-water systems, irrespective of their water solubilities, no (re)crystallization of water at lower $W_{\mathrm{C}}$ values can be easily understood from a physicochemical point of view. As expected, in all of the cases (including PEG) cited above, no (re)crystallization of water has been demonstrated at lower $W_{\mathrm{C}}$ values. Except for the PEG-water system, with an increase of $W_{\mathrm{C}}$, the total amount of (re)crystallized water increased monotonously, as was easily predicted. As seen in Figure 1, on the other hand, the PEG-water system demonstrated the reappearance of a $W_{\mathrm{C}}$ at which no (re)crystallization of water occurred, while the total amount of (re)crystallized water increased. It is noteworthy that recrystallized water existed below and above the $W_{\mathrm{C}}$ at which perfect vitrification occurred. The reason for the occurrence of perfect vitrification has not been satisfactorily explained in the literature. If the hydration structure of recrystallizable water is assumed to be the same regardless of the type of polymer and $W_{\mathrm{C}}$ values as per the hypothesis mentioned above, it is difficult to explain this phenomenon.

Our recent study using a PEG aqueous solution with a $W_{\mathrm{C}}$ of $37.5 \mathrm{wt} \%$ revealed that the recrystallizable water in this solution had two different types of hydration structures and that their recrystallization temperatures were different. ${ }^{54}$ The perfect vitrification of the PEG-water system was probably related to the existence of these two different types of hydration structure. In the present study, to reveal the cause of the perfect vitrification, the thermal properties of PEG aqueous solutions with various $W_{\mathrm{C}}$ values in the range $\sim 36-51 \mathrm{wt} \%$ were investigated by temperature-variable Fourier-transform mid-infrared spectroscopy (TVFT-IR). Unlike calorimetric methods such as DSC, infrared (IR) spectroscopy probing a molecular vibration cannot detect heat fluxes in state changes of matter via temperature change but does provide information about molecular orientation and conformation in situ.

\section{Experiments}

Poly(ethylene glycol) dimethyl ether (PEG) (Aldrich) was purified via precipitation in ethanol-chloroform. The weight-average and number-average molecular weights of PEG were 2064 and 2036, respectively. The quality of water used in all experiments was Milli-Q grade (resistivity $>18.2 \mathrm{M} \Omega \cdot \mathrm{cm}$ ). PEG aqueous solutions with seven different $W_{\mathrm{C}}$ values of 36.4, 38.6, 40.7, 43.3, 45.1, 46.1, and $50.9 \mathrm{wt} \%$ were prepared. Each $W_{\mathrm{C}}$ value is expressed as the percentage of the weight of water over the weight of the total solution.

Mid-IR spectra of the PEG aqueous solutions were recorded on a Spectrum One NTS (Perkin-Elmer) with a resolution of $4 \mathrm{~cm}^{-1}$ and a scanning time of $20 \mathrm{~s}$ over a temperature $(T)$ range of $298-170 \mathrm{~K}$. The cooling rate was either 1.0 or $4.8 \mathrm{~K} \cdot \mathrm{min}^{-1}$, and the heating rate was $1.0 \mathrm{~K} \cdot \mathrm{min}^{-1}$. The temperature of the sample was controlled with an Optistate-DN cryostat (Oxford) connected to a model ITC601 $T$ controller. An appropriate amount of PEG aqueous solution, typically $1 \mu \mathrm{L}$, was placed between two sapphire substrates (13 mm in diameter and $1 \mathrm{~mm}$ in thickness), and the sapphire cell was hermetically sealed with indium. The cell length (thickness of the sample) was not determined, but it was confirmed that the maximum absorbance in the observed wavenumber range $\left(4000-2500 \mathrm{~cm}^{-1}\right)$ was below 1 at room $T$.

\section{Results}

3.1. Room Temperature (Figure 2). Mid-IR spectra of PEG aqueous solutions with various $W_{\mathrm{C}}$ values at 298 $\mathrm{K}$ are shown in Figure 2. The spectral intensity was normalized at $2881 \mathrm{~cm}^{-1}$ (Figure 2A).

3.1.1. PEG. Several components that overlapped with each other in the wavenumber range of 3000-2800 $\mathrm{cm}^{-1}$ (Figure 2B) were C-H stretching vibrations of the PEG chain. Among them, the component at $\sim 2918 \mathrm{~cm}^{-1}$ was a $\mathrm{C}-\mathrm{H}$ stretching vibration of $\mathrm{PEG}$ with a random-coil conformation, and the component at $\sim 2881 \mathrm{~cm}^{-1}$ was an overlapped one of the $\mathrm{C}-\mathrm{H}$ stretching vibrations of $\mathrm{PEG}$ with random-coil and "tgt" conformations. ${ }^{16-21}$ For the tgt conformation, the sequence $-\mathrm{O}-\mathrm{CH}_{2}-\mathrm{CH}_{2}-\mathrm{O}-$ had a trans $(t)$ conformation about the $-\mathrm{O}-\mathrm{C}-$ bond, a gauche $(g)$ conformation about the $-\mathrm{C}-\mathrm{C}-$ bond, and a $t$ conformation about the $-\mathrm{C}-\mathrm{O}-$ bond. The tgt conformation was a basic unit of a $7 / 2$ helical segment formed in the crystalline solid PEG (dotted spectrum in Figure 2B). Following the literature on PEG aqueous solutions at room temperature, a portion of the PEG chain had the 7/2 helical conformation in the aqueous solution. A decrease in the ratio of absorbance at $2881 \mathrm{~cm}^{-1}$ (A2881) and $2918 \mathrm{~cm}^{-1}$ (A2918), that is, A2881/A2918, with $W_{\mathrm{C}}$ (Figure 2C) indicated a relative decrease of the helical PEG segment.

3.1.2. Water. The mid-IR spectra of water in the PEG aqueous solutions had at least three components: two shoulders at 3520 and $3270 \mathrm{~cm}^{-1}$ and a peak at $3430 \mathrm{~cm}^{-1}$. Pure water (dotted spectrum in Figure 2A) also had at least these three components in its spectrum, but the three components differed from those of the PEG solutions: two shoulders at 3650 and $3240 \mathrm{~cm}^{-1}$ and a peak at $3400 \mathrm{~cm}^{-1}$. As seen in the spectra normalized over the high wavenumber region (Figure 2D), the relative intensity of the spectra in the low wavenumber region increased with $W_{\mathrm{C}}$. The ratio of 
integrated absorbance in the $\mathrm{O}-\mathrm{H}$ stretching region $\left(\mathrm{OH}_{\text {area }}, 3700-3000 \mathrm{~cm}^{-1}\right)$ to that in the $\mathrm{C}-\mathrm{H}$ stretching region $\left(\mathrm{CH}_{\text {area }}, 3000-2700 \mathrm{~cm}^{-1}\right)$ showed a slight quadratic increase with $W_{\mathrm{C}}$ (Figure $2 \mathrm{E}$ ), suggesting that the number of water molecules hydrogen-bonded between other water molecules increased with the $W_{\mathrm{C}}$. The numbers of water molecules per repeat unit of PEG $\left(N_{\text {water }}\right)$ at the highest $(50.9 \mathrm{wt} \%)$ and lowest $(36.4 \mathrm{wt} \%) W_{\mathrm{C}}$ values in this study were 2.54 and 0.399 , respectively. Several studies showed that the number of bound water molecules per repeat unit of PEG was in the range of 1-4. If the term "bound water" indicates water molecules that strongly interacted with the PEG chain, it can be said that hydrogen bonds between water molecules existed but that no water interacted without the PEG chain. A molecular-dynamics simulation also showed no free water at $W_{\mathrm{C}}<50 \mathrm{wt} \%{ }^{25}$

3.2. Temperature dependence. For basic information concerning pure water, the temperature dependence of the spectrum of pure water is shown in Figure 3. The top and bottom panels of the figure show the temperature dependence of the spectrum of water and that of the absorbance at $3250 \mathrm{~cm}^{-1}$ (A3250), respectively, and the left and right panels show the results for the cooling and heating processes, respectively. At $251 \mathrm{~K}$ during cooling, the spectrum with a large width suddenly changed to one with a very sharp peak at $\sim 3250 \mathrm{~cm}^{-1}$. The opposite change was observed at $273 \mathrm{~K}$ during heating. The two changes indicated crystallization and fusion, respectively. The A3250 value at the two state changes sharply increased and decreased, respectively. The two state changes should be judged on the basis of changes in the spectra and A3250 values.

The results for PEG aqueous solutions in the temperature range of $298-170 \mathrm{~K}$ at $W_{\mathrm{C}}$ values of 38.6 , 43.2, and 50.8 wt $\%$ are shown in Figures 4, 5, and 6, respectively. The spectra of the other $W_{\mathrm{C}}$ values are given in the Supporting Information. The results at $W_{\mathrm{C}}$ values of $38.6 \mathrm{wt} \%$ (Figure 4) and $50.8 \mathrm{wt} \%$ (Figure 6 ) are representative cases for those below and above a $W_{\mathrm{C}}$ of $43.2 \mathrm{wt} \%$, respectively. Panels (A) and (B) in each figure show the spectra obtained at a cooling rates of $4.8 \mathrm{~K} \cdot \mathrm{min}^{-1}$ (rapid cooling) and $1.0 \mathrm{~K} \cdot \mathrm{min}^{-1}$ (slow cooling), respectively, and the top and bottom parts in each panel are spectra of the $\mathrm{O}-\mathrm{H}$ stretching and $\mathrm{C}-\mathrm{H}$ stretching regions, respectively. Panel (C) shows the temperature dependence of the value of A3250 (top) and that of A2881/A2918 (bottom). The left and right panels show the results of the cooling and heating processes, respectively.

3.2.1. $W_{\mathbf{C}}<43.2 \mathrm{wt} \%(\mathbf{3 8 . 6} \mathbf{w t} \%$, Figure 4). Irrespective of the cooling rate, the A2881/A2918 value increased following a saturation curve with cooling until $240 \mathrm{~K}$ (bottom of Figure $4 \mathrm{C}$ ). In the case of rapid cooling, a slight decrease in the A2881/A2918 value was observed below $240 \mathrm{~K}$, but a drastic change was not observed. In the case of slow cooling, on the other hand, a sharp increase of the value was observed, indicating crystallization of the PEG, and was confirmed by the similar shape of the spectrum to that of the crystalline solid PEG (bottom of Figure 4B). At the same $T(\sim 240 \mathrm{~K})$, a sharp increase of the A3250 value was also observed (left top of Figure 4C). The spectra in the O-H stretching region below $\sim 240 \mathrm{~K}$ were similar to the spectrum of ice (left top of Figure $4 \mathrm{~B}$ ), indicating ice formation. In the case of rapid cooling, the slight deviation from the change of the A3250 value (left top of Figure 4C) might indicate a small amount of ice formation, although an ice-like spectrum was not clearly observed (left top of Figure 4A).

During heating, in the case of the sample treated by slow cooling (Figure 4B), the temperature at which steep changes were observed was $\sim 260 \mathrm{~K}$ and differed from that during cooling $(240 \mathrm{~K})$, but the changes of the spectrum and the values of A3250 and A2881/A2918 were the same as those of the cooling process in the opposite direction. In the case of the sample prepared by rapid cooling, steep increases in the values of A3250 and A2885/A2918 were observed in the temperature range of 198-220 K (right bottom of Figure 4C), indicating recrystallizations of both water and PEG. The difference from crystallization was a two-step event, and a detailed discussion of this two-step recrystallization has been reported previously. ${ }^{54}$ In brief, the increase in A3250 in the low-temperature range of 198-210 K was comparable to that in the high temperature range of $210-220 \mathrm{~K}$, whereas the increase in A2881/A2918 in the lower temperature range was approximately three-times less than that in the higher temperature range. This indicates that the water molecules that crystallized in the lower and higher temperature ranges corresponded to water molecules associated with the PEG chains with helical and random-coil conformations, respectively. The former and latter water molecules are called "type-H" and "type-R" water molecules, respectively. Above $230 \mathrm{~K}$, the observed changes were the same, irrespective of the cooling rate; the values of A3250 and A2885/A2918 decreased steeply at $260 \mathrm{~K}$ and then returned to their initial values.

3.2.2. $W_{\mathrm{C}}=43.2 \mathrm{wt} \%$ (Figure 5). The spectral changes of the samples treated by both slow cooling and rapid cooling were almost the same, and the comparable panel (B) is therefore omitted. As seen in the spectra, in the observed temperature range, an ice-like spectrum was not observed in either the cooling or heating process. The values of A3250 and A2881/A2918 also did not show steep or stepped changes, such as would suggest state changes of water and PEG (crystallization and recrystallization). This phenomenon was a perfect vitrification, that is, neither crystallization nor recrystallization occurred. The other difference from the samples below a $W_{\mathrm{C}}$ of $43.2 \mathrm{wt} \%$ was the change of A2885/A2918 with cooling; the value at a $W_{C}$ of $43.2 \mathrm{wt} \%$ decreased (left bottom of Figure 5C), whereas those below a $W_{\mathrm{C}}$ of $43.2 \mathrm{wt} \%$ increased (left bottom of Figure $5 \mathrm{C}$ ).

3.2.3. $W_{\mathrm{C}}>43.2 \mathrm{wt} \%(50.8 \mathrm{wt} \%$, Figure 6). Spectral changes at both rapid cooling and slow cooling were similar to those at $W_{\mathrm{C}}$ values of $43.2 \mathrm{wt} \%$ and $38.6 \mathrm{wt} \%$, respectively, and the comparable panels (A) and (B) are therefore omitted. In the cooling process, no ice-like spectra were clearly observed. For the slow cooling, however, a 
stepped increase in the A3250 value was observed at $230 \mathrm{~K}$, suggesting crystallization of a small amount of water molecules. The A2881/A2918 value increased synchronously with A3250, indicating crystallization of PEG. A gradual change in the values of A3250 and A2881/A2918 in the observed temperature range for rapid cooling indicated no crystallization of water or PEG, that is, vitrification. A steep increase in both the values of A3250 and A2881/A2918 in the heating process was observed at $\sim 220 \mathrm{~K}$, indicating recrystallization of both water and PEG. Recrystallization above a $W_{\mathrm{C}}$ of $43.2 \mathrm{wt} \%$ was a one-step event, unlike that of solutions with a $W_{\mathrm{C}}$ below $43.2 \mathrm{wt} \%$.

\section{Discussion}

The state changes of water and PEG via temperature perturbation observed spectroscopically are summarized in Table 1. Except for a solution with a $W_{\mathrm{C}}$ of $43.2 \mathrm{wt} \%$, crystallization and/or recrystallization (and fusion) occurred for both water and PEG. Perfect vitrification was observed at a $W_{\mathrm{C}}$ of $43.2 \mathrm{wt} \%$. This specific $W_{\mathrm{C}}$ is expressed as " $W_{\mathrm{CPV}}$ " hereafter. The crystallization $T(\sim 237 \mathrm{~K})$ below $W_{\mathrm{CPV}}$ was $\sim 10 \mathrm{~K}$ greater than that above $W_{\mathrm{CPV}}(\sim 226 \mathrm{~K})$. The two-step recrystallization was not observed above $W_{\mathrm{CPV}}$, and their recrystallization $T$ values $(\sim 213 \mathrm{~K})$ were similar to the $T$ value of the two-step recrystallization below $W_{\mathrm{CPV}}(\sim 210 \mathrm{~K})$. DSC measurements also demonstrated perfect vitrification at a specific $W_{\mathrm{C}}$, two-step recrystallization in the lower $W_{\mathrm{C}}$ range, and difference in the $T$ values of each state change that depended on $W_{\mathrm{C}}{ }^{34-37}$ Some questions, however, have remained. How can the difference in crystallization $T$ values below and above $W_{\mathrm{CPV}}$ be explained? What is the difference between the one-step and two-step recrystallization processes above and below $W_{\mathrm{CPV}}$, respectively? Why does perfect vitrification occur at this specific $W_{\mathrm{C}}$ ?

The state changes via temperature perturbation observed spectroscopically, except for crystallization, recrystallization, and fusion, were related to the relative amount of the helical segment of PEG (the value of A2881/A2918); the amount (the value) at $W_{\mathrm{C}} \geq W_{\mathrm{CPV}}$ decreased and that at $W_{\mathrm{C}}<W_{\mathrm{CPV}}$ increased (Table 1). It can be said that at the former and latter $W_{\mathrm{C}}$ ranges, the amounts of type-H water molecules decreased and increased with cooling, respectively. If it can be assumed that type-H and type- $\mathrm{R}$ water molecules had limiting concentrations for their (re)crystallization, this assumption could provide plausible explanations for the questions listed above.

The crystallization $T$ below $W_{\mathrm{CPV}}$ was $\sim 10 \mathrm{~K}$ greater than that above $W_{\mathrm{CPV}}$. Our previous study demonstrated that during two-step recrystallization, type-H water molecules recrystallized at a lower $T(\sim 198 \mathrm{~K})$ than did type-R water molecules $(\sim 210 \mathrm{~K})$, indicating that the interaction of the former with the PEG chains was weaker than that of the latter. In the cooling process, the type-H water molecules might have crystallized at a higher $T$ than did the type- $\mathrm{R}$ water molecules. ${ }^{50}$ If the concentration of type-H water molecules was less than the limiting concentration for their crystallization, the crystallization $T$ might have been dominated by type- $\mathrm{R}$ water molecules. Below $W_{\mathrm{CPV}}$, the relative amount of the helical segment steeply increased with cooling, resulting in an increase in the amount of type-H water molecules. These type-H water molecules were considered to be crystallized. However, two-step crystallization (such as recrystallization) expected from crystallization of type- $\mathrm{R}$ water molecules was not observed. Crystallization of type- $\mathrm{H}$ water molecules should include a phase separation with the formation of a crystalline solid PEG. Differing from an intramolecular conformational transition such as the coil-to-helix transition, the phase separation should be accompanied by a dynamic (macroscopic) molecular diffusion/rearrangement. This dynamic transition likely triggers the coil-to-helix transition of the remaining random-coil segments. As a result, during crystallization of type-H water molecules, the amount of type- $\mathrm{R}$ water molecules decreased and their crystallization was not observed.

Recrystallization above and below $W_{\mathrm{CPV}}$ occurred in one and two steps, respectively. As mentioned repeatedly, during the two-step recrystallization, type-H and type- $\mathrm{R}$ water molecules recrystallized in the first (198 K) and second $(210 \mathrm{~K})$ steps, respectively. This phenomenon conflicts with the aforementioned discussion of crystallization; if the discussion could be adapted to the recrystallization phenomenon, recrystallization should occur in one step. This discrepancy can be explained by considering that the glass transition $T\left(T_{\mathrm{g}}\right)$ of the hydrated helical segment was less than that of the hydrated random-coil segment. At the $T$ at which type-H water molecules recrystallized, the motion of type-R water molecules in a glassy state might be restricted, and their recrystallization, which requires molecular diffusion/rearrangement, could not occur. This conclusion is consistent with the fact that the recrystallization $T$ values $(198 \mathrm{~K}$ and $210 \mathrm{~K})$ were sufficiently less than the crystallization $T$ values $(237 \mathrm{~K}$ and $226 \mathrm{~K})$. This consideration, however, is not sufficient to explain the perfect vitrification.

Perfect vitrification was observed at a $W_{\mathrm{C}}$ of $43.2 \mathrm{wt} \%\left(W_{\mathrm{CPV}}\right)$. Based on the aforementioned assumption-that the type-H and the type- $\mathrm{R}$ water molecules had limiting concentrations for their (re)crystallization-an explanation for the perfect vitrification should be easily obtained. At $W_{\mathrm{CPV}}$, both the concentrations of type-H and type- $\mathrm{R}$ water molecules were less than their limiting concentrations. However, such a result meant that at $W_{\mathrm{C}}<W_{\mathrm{CPV}}$, the concentration of type-R water molecules should have been less than their limiting concentration and, therefore, one-step recrystallization at $W_{\mathrm{C}}<W_{\mathrm{CPV}}$ should have occurred. This is clearly inconsistent with the actual experimental results. This discrepancy might be solved by considering the local concentration. Recrystallization of type-H water molecules and PEG resulted from the phase separation of ice and solid PEG. At the same time, this phase separation also indicated phase separation of the hydrated random-coil chains of PEG, including the type-R water molecules, namely, the local concentration of type-R water molecules was increased, meaning that the type-R water molecules could be recrystallized above the $T_{\mathrm{g}}$ of the hydrated random-coil domain. 


\section{Conclusion}

Temperature-variable measurements of the mid-IR spectra of PEG aqueous solutions with various $W_{\mathrm{C}}$ values demonstrated the existence of a specific $W_{\mathrm{C}}, 43.2 \mathrm{wt} \%$, at which no (re)crystallization of water or PEG in a temperature range of 298-170 K occurred: perfect vitrification. At the other $W_{\mathrm{C}}$ values, vitrification during cooling was observed, but a large amount of recrystallization (devitrification) also occurred. These phenomena were caused by the existence of two types of water molecules that were associated with the PEG chains with helical and random-coil segments. The assumption that the two types of water molecules might have limiting concentrations for their (re)crystallization provided a plausible explanation for the perfect vitrification.

Acknowledgements. This work was supported by Grants-in-Aid for Scientific Research (Nos. 26288100 and 15K04604) from JSPS. The authors are indebted to Messrs. T. Motonaga and R. Kasai for their technical assistance.

Supporting Information Available. $T$ dependence of spectra of the PEG aqueous solutions with water contents of $36.4,40.7,43.2,45.1,46.1$, and $50.9 \mathrm{wt} \%$. This material is available free of charge via the Internet at http://pubs.acs.org.

\section{References}

(1) Prime, K. L.; Whitesides, G. M. Adsorption of Proteins onto Surfaces Containing End-Attached Oligo(ethylene oxide): A model System Using Self-Assembled Monolayers. J. Am. Chem. Soc. 1993, 115, 10714-10721.

(2) Prime, K. L.; Whitesides, G. M. Self-Assembled Organic Monolayers: Model Systems for Studing Adsorption of Proteins at Surfaces. Science 1991, 252, 1164-1167.

(3) Chapman, R. G.; Ostuni, E.; Takayama, S.; Holmlin, R. E.; Yan, L.; Whitesides, G. M. Surveying for Surfaces that Resist the Adsorption of Proteins. J. Am. Chem. Soc. 2000, 122, 8303-8304.

(4) Ostuni, E.; Chapman, R. G.; Holmlin, R. E.; Takayama, S.; Whitesides, G. M. A Survey of Structure-Property Relationships of Surfaces that Resist the Adsorption of Protein. Langmuir 2001, 17, 5605-5620.

(5) Ostuni, E.; Grzybowski, B. A. Mrksich, M.; Roberts, C. S.; Whitesides, G. M. Adsorption of Proteins to Hydrophobic Sites on Mixed Self-Assembled Monolayers. Langmuir 2003, 19, 1861-1872.

(6) Meyers, S. R.; Grinstaff, M. W. Biocompatible and Bioactive Surface Modifications for Prolonged In Vivo Efficacy. Chem. Rev. 2011, 112, 1615-1632.

(7) Morra, M. In Water in Biomaterials Surface Science; Morra, M., Ed.; Wiley: Chichester, 2001, p.307-332.

(8) Zolk, M.; Eisert, F.; Pipper, J.; Herrwerth, S.; Eck, W.; Buck, M.; Grunze, M. Solvation of Oligo(ethylene glycol)-Terminated Self-Assembled Monolayers Studied by Vibrational Sum Frequency Spectroscopy. Langmuir 2000, 16, 5849-5852.

(9) Harder, P.; Grunze, M.; Dahint, R.; Whitesides, G. M.; Laibinis, P. E. Molecular Conformation in Oligo(ethylene glycol)-Terminated Self-assembled Monolayers on Gold and Silver Surfaces Determines Their Ability to Resist Protein Adsorption. J. Phys. Chem. B 1998, 102, 426-436.

(10) Wang, R. L. C.; Kreuzer, H. J.; Grunze, M. Molecular Conformation and Solvation of Oligo(ethylene glycol)-Terminated Self-assembled Monolayers and Their Resistance to Protein Adsorption. J. Phys. Chem. B 1997, 101, 9767-9773.

(11) Pertsin, A. J.; Grunze, M. Computer Simulation of Water near the Surface of Oligo(ethylene glycol)-Terminated Alkanethiol Self-Assembled Monolayers. Langmuir 2000, 16, 8829-8841.

(12) Corn, R. M.; Higgins, D. A. Optical Second Harmonic Generation As a Probe of Surface Chemistry. Chem. Rev. 1994, 94, 107-125.

(13) Blandamer, M. J.; Fox, M. F.; Powell, E.; Stafford, J. W. A Viscometric Study of Poly(ethylene oxide) in t-Butyl Alcohol/Water Mixtures. Makromol. Chem. 1969, 124, 222-231.

(14) Bailey, F. E. Jr.; Callard, R. W. Some Properties of Poly(ethylene oxide) in Aqueous Solution. J. Appl. Polym. Sci. 1959, 1, 56-62.

(15) Bailey, F. E. Jr.; Callard, R. W. Thermodynamic Parameters Some Properties of Poly(ethylene oxide) in Aqueous Solution. J. Appl. Polym. Sci. 1959, 1, 373-374.

(16) Borodin, O.; Trouw, F.; Bedrov, D.; Smith, G. D. Temperature Dependence of Water Dynamics in Poly(Ethylene Oxide)/Water Solutions from Molecular Dynamics Simulations and Quasielastic Neutron Scattering Experiments. J. Phys. Chem. B 2002, 106, 5184-5193.

(17) Trouw, F. R.; Borodin, O.; Cook, J. C.; Copey, J. R. D.; Smith, G. D. Quasielastic Neutron-Scattering Study of the Local Dynamics of Poly(ethylene glycol) Dimethyl Ether in Aqueous Solution. J. Phys. Chem. B 2003, 107, 10446-10452.

(18) Connor, T. M.; McLauchlan, K. A. High Resolution Nuclear Resonance Studies of the Chain Conformation of Polyethylene Oxide. J. Phys. Chem. 1965, 69, 1888-1893.

(19) Maeda, Y.; Ide, M.; Kitano, H. Vibrational spectroscopic study on the structure of water in polymer systems. J. Mol. 
Liquid 1999, 80, 149-163.

(20) Wada, R.; Fujimoto, K.; Kato, M. Why Is Poly(oxyethylene) Soluble in Water? Evidence from the Thermodynamic Profile of the Conformational Equilibria of 1,2-Dimethoxyethane and Dimethoxymethane Revealed by Raman Spectroscopy J. Phys. Chem. B 2014, 118, 12223-12231.

(21) Wahab, S. A.; Matsuura, H. Raman Spectroscopic Study of Conformational Properties of a Short-Chain Poly(oxyethylene)(C1E3C1) in Polar Solvents at Different Temperature. J. Mol. Struct. 2002, 606, 35-43.

(22) Liu, K.; Parsons, J. L. Solvent Effects on the Preferred Conformation of Poly(ethylene glycols). Macromolecules 1969, 2, 529-533.

(23) Begum R.; Matsuura, H. Conformationl Properties of Short Poly(oxyethylene) Chains in Water Studied by IR Spectroscopy. J. Chem. Soc., Faraday Trans. 1997, 93, 3839-3848.

(24) Ide, M.; Yoshikawa, D.; Maeda, Y.; Kitano, H. State of Water Inside and at the Surface of Poly(ethylene glycol) Films Examined by FT-IR. Langmuir 1999, 15, 926-929.

(25) Smith, G. D.; Bedrov, D.; Borodin, O. Molecular Dynamics Simulation Study of Hydrogen Bonding in Aqueous Poly(ethylene oxide) Solutions. Phys. Rev. Lett. 2000, 85, 5583--5586.

(26) Smith, G. D. Bedrov, D.; Borodin O. Conformations and Chain Dimensions of Poly(ethylene oxide) in Aqueous Solution: A Molecular Dynamics Simulation Study. J. Am. Chem. Soc. 2000, 122, 9548-9549.

(27) Tasaki, K. Poly(oxyethylene)-Water Interactions: A Molecular Dynamics Study. J. Am. Chem. Soc. 1996, 8459-8469.

(28) Smith, G. D.; Bedrov, D. A Molecular Dynamics Simulation Study of the Influence of Hydrogen-Bonding and Polar Interactions on Hydration and Conformations of a Poly(ethylene oxide) Oligomer in Dilute Aqueous Solution. Macromolecules 2002, 35, 5712-5719.

(29) Fischer, J.; Paschek, D.; Geiger, A.; Sadowski, G. Modeling of Aqueous Poly(oxyethylene) Solutions: 1. Atomistic Simulations J. Phys. Chem. B 2008, 112, 2388-2398.

(30) Fischer, J.; Paschek, D.; Geiger, A.; Sadowski, G. Modeling of Aqueous Poly(oxyethylene) Solutions. 2. Mesoscale Simulations. J. Phys. Chem. B 2008, 112, 13561-13571.

(31) Wahab, S. A.; Harada, T.; Matsubara, T.; Aida, M. Quantum Chemical Study of the Interaction of the Sort-Chain Poly(oxyethylene)s $\mathrm{CH}_{3}\left(\mathrm{OCH}_{2} \mathrm{CH}_{2}\right)_{m} \mathrm{OCH}_{3}\left(\mathrm{C}_{1} \mathrm{E}_{m} \mathrm{C}_{1} ; m=1\right.$ and 2) with a Water Molecule in the Gas Phase and in Solutions. J. Phys. Chem. A 2006, 110, 1052-1059.

(32) Aray, Y.; Marquez, M.; Rodrguez, J.; Vega, D.; Simon-Manso, Y.; Coll, S.; Gonzalez, C.; Weitz, D. A. Electrostatics for Exploring the Nature of the Hydrogen Bonding in Polyethylene Oxide Hydration. J. Phys. Chem. B 2004, 108, 2418-2424.

(33) Dormidontova, E. E. Role of Competitive PEO-Water Hydrogen Bonding in Aqueous Solution PEO Behavior. Macromolecules 2002, 35, 987-1001.

(34) Huang, L.; Nishinari, K. Interaction Between Poly(ethylene glycol) and Water as Studied by Differential Scanning Calorimetry. J. Polym. Sci. B: Polym. Phys. 2001, 39, 496-506.

(35) Hatakeyama, T.; Kasuga, H.; Tanaka, M.; Hatakeyama, H. Cold Crystallization of Poly(ethylene glycol)-Water systems. Thermochim. Acta 2007, 465, 59-66.

(36) Kimizuka, N.; Viriyarattanasak, C.; Suzuki, T. Ice Nucleation and Supercooling Behavior of Polymer Aqueous Solutions. Cryobiology 2008, 56, 80-87.

(37) Takei, T.; Sugitani, Y. High Frequency Spectroscopic Study of the Bound State of Water in PEG- $\mathrm{H}_{2} \mathrm{O}$ System during Heating from Frozen State. Anal. Sci. 2010, 26, 337-341.

(38) de Vringer, T.; Joosten, J. G.; Junginger, H. E. A Study of the Hydration of Polyoxyethylene at Low Temperatures by Differential Scanning Calorimetry. Colloid Polym. Sci. 1986, 264, 623-630.

(39) Tilcock, C. P. S.; Fisher, D. The Interaction of Phospholipid Membranes with Poly(ethylene glycol). Vesicle Aggregation and Lipid Exchange. Biochim. Biophys. Acta 1982, 688, 645-652.

(40) Kjellander, R.; Florin, E. Water Structure and Changes in Thermal Stability of the System Poly(ethylene oxide) -Water. J. Chem. Soc., Faraday Trans. 1 1981, 77, 2053-2077.

(41) Hager, S. L.; Macrury, T. B. Investigation of Phase Behavior and Water Binding in Poly(alkylene oxide) Solutions. J. Appl. Polym. Sci. 1980, 25, 1559-1571.

(42) Furushima, Y.; Ishikiriyama, K.; Ueno, Y.; Sugaya, H. Analysis of the Water in Polyvinylpyrrolidone Aqueous Solution Using DSC Method. Thermochim. Acta 2012, 538, 43-47.

(43) Hatakeyama, T.; Naoi, S.; Hatakeyama, H. Phase Transition of Locust Bean Gum-, Tara Gum- and Guar Gum-Water Systems. J. Therm. Anal. Calorim. 2002, 70, 841-852.

(44) Yoshida, H.; Hatakeyama, T.; Hatakeyama, H. Phase-transitions of the Water-Xanthan System. Polymer 1990, 31, 693-698.

(45) Hatakeyama, T.; Yoshida, H.; Hatakeyama, H. A Differential Scanning Calorimetry Study of the Phase Transition 
of the WateSodium Cellulose Sulfate System. Polymer 1987, 28, 1282-1286.

(46) Franks, F. In "Water: A Comprehensive Treatise"; Franks, F. Ed.; Plenum Press: New York, 1982; Vol. 7.

(47) Green, J. L.; Fan, J.; Angell, C. A. The Protein-glass Analogy: New Insight From Homopeptide Comparisons. J. Phys. Chem. 1994, 98, 13780-13790.

(48) Tanaka, M.; Motomura, T.; Kawada, M.; Anzai, T.; Kasori, Y.; Shiroya, T.; Shimura, K.; Onishi, M.; Mochizuki, A. Blood Compatible Aspects of Poly(2-methoxyethylacrylate) (PMEA) - Relationship between Protein Adsorption and Platelet Adhesion on PMEA Surface. Biomaterials 2000, 21, 1471-1481.

(49) Tanaka, M.; Motomura, T.; Ishii, N.; Shimura, K.; Onishi, M.; Mochizuki, A.; Hatakeyama, T. Cold Crystallization of Water in Hydrated Poly(2-methoxyethylacrylate) (PMEA). Polym. Int. 2000, 49, 1709-1713.

(50) Tanaka, M.; Mochizuki, A.; Ishii, N.; Motomura, T.; Hatakeyama, T. Study of Blood Compatibility with Poly(2-methoxyethyl acrylate). Relationship between Water Structure and Platelet Compatibility in Poly(2-methoxyethylacrylate-co-2-hydroxyethylmethacrylate). Biomacromolecules 2002, 3, 36-41.

(51) Gemmei-Ide, M.; Ohya, A.; Kitano, H. Recrystallization of Water in Non-Water-Soluble (Meth)Acrylate Polymers Is Not Rare and Is Not Devitrification. J. Phys. Chem. B 2012, 116, 1850-1857.

(52) Gemmei-Ide, M.; Kitano, H. Spectroscopic Evidence of Phase Transition of Monomolecular Water in Solid Polystyrene. J. Phys. Chem. B 2008, 112, 2764-2766.

(53) Gemmei-Ide, M.; Kitano, H. Crystal Growth of Ice Ih by Revapor-Deposition and Diffusion Suppression of Monomolecular Water in a Polymer Soild: Spectroscopic Observation of Phase Transition of Water Sorbed into Solid Polystyrene. J. Phys. Chem. B 2008, 112, 13499-13502.

(54) Gemmei-Ide, M.; Motonaga, T.; Kasai, R.; Kitano, H. Two-Step Recrystallization of Water in Concentrated Aqueous Solution of Poly(ethylene glycol). J. Phys. Chem. B 2013, 117, 2188-2194. 
Table 1. Crystallization, recrystallization, and melting temperatures.

\begin{tabular}{|c|c|c|c|c|c|c|c|}
\hline \multirow{2}{*}{$\begin{array}{c}W_{\mathrm{C}} \\
/ \mathrm{wt} \%\end{array}$} & \multicolumn{2}{|c|}{$\begin{array}{c}\text { Crystallization } \\
\text { / K }\end{array}$} & \multicolumn{2}{|c|}{$\begin{array}{c}\text { Recrystallization } \\
\text { / K }\end{array}$} & \multicolumn{2}{|c|}{$\begin{array}{c}\text { Melting } \\
\text { / K }\end{array}$} & \multirow{2}{*}{$\begin{array}{c}\text { Change direction of } \\
\text { A2881/A2918 } \\
\text { during cooling to } 250 \mathrm{~K}\end{array}$} \\
\hline & Water & PEG & Water & PEG & Water & PEG & \\
\hline$<43.2$ & \multicolumn{2}{|c|}{237} & $198(210)$ & $210(198)$ & \multicolumn{2}{|c|}{260} & Increase \\
\hline$=43.2$ & \multicolumn{2}{|c|}{ N.D. } & \multicolumn{2}{|c|}{ N.D. } & \multicolumn{2}{|c|}{ N.D. } & Decrease \\
\hline$>43.2$ & \multicolumn{2}{|c|}{226} & \multicolumn{2}{|c|}{213} & & & Decrease \\
\hline
\end{tabular}

N.D.: Not detected. 

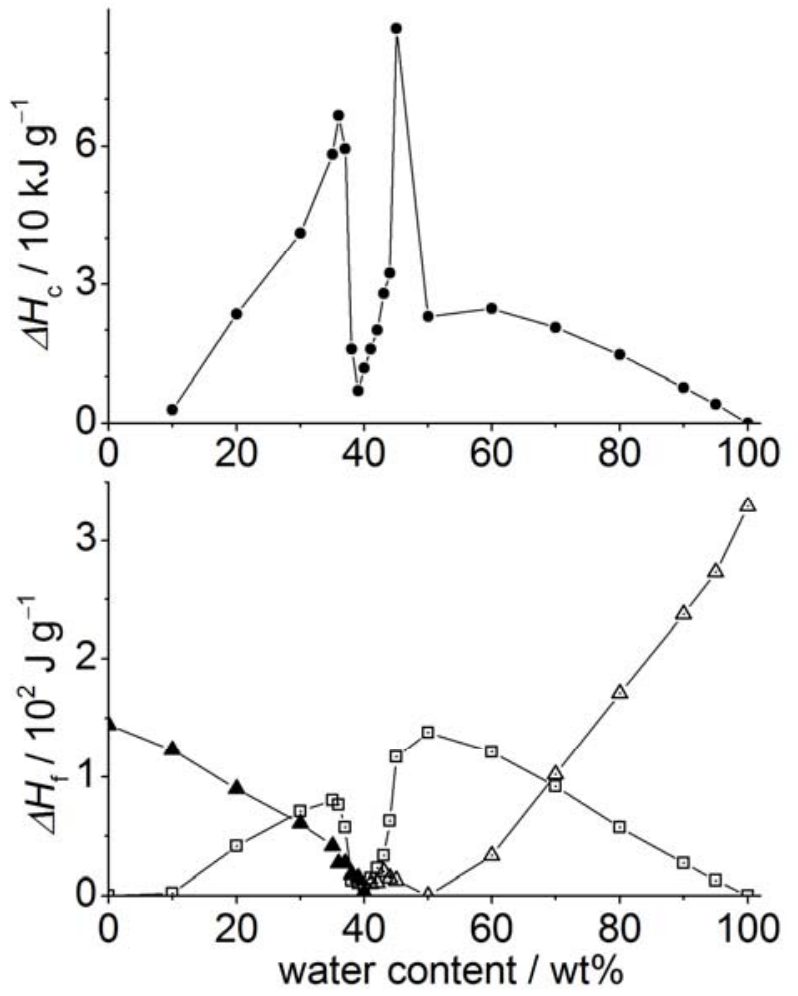

Figure 1. Recrystallization enthalpies $(\bullet)$ and fusion enthalpies of PEG $(\triangleleft)$, a eutectic mixture of PEG-water $(\square)$, and water $(\triangle)$ observed for PEG aqueous solutions with various $W_{C}$ values, determined by DSC measurements. The molecular weight of PEG was 1540 . The figure was redrawn from the work of Huang et al. ${ }^{36}$ 

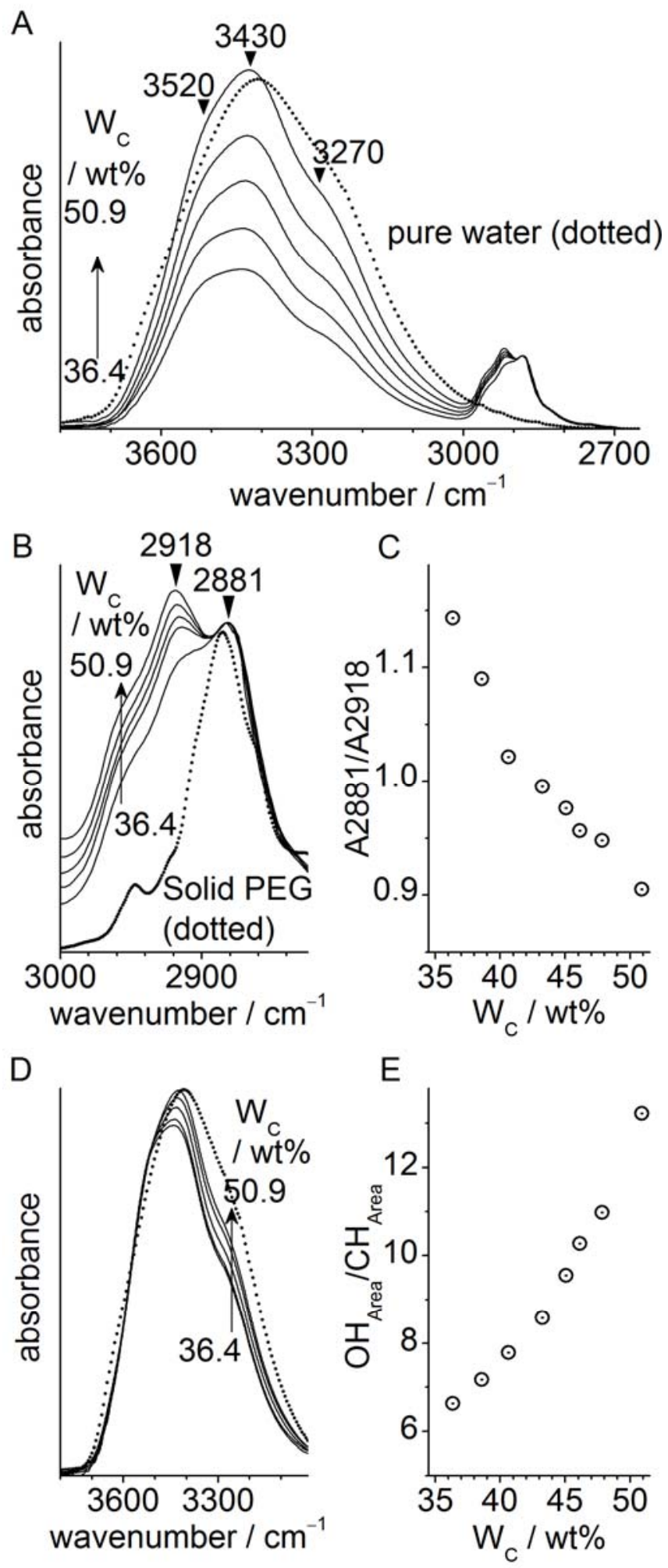

Figure 2. Mid-IR spectra of PEG aqueous solutions with various water contents $\left(W_{\mathrm{C}}\right)$ at $298 \mathrm{~K}$. (A) Selected spectra in the wavenumber range of $3800-2650 \mathrm{~cm}^{-1}$. Spectral intensity was normalized at $2885 \mathrm{~cm}^{-1}$. (B) Spectra of the $\mathrm{C}-\mathrm{H}$ stretching region $\left(3000-2800 \mathrm{~cm}^{-1}\right)$. (C) $W_{C}$ dependence of the A2881/A2918 value. (D) Spectra of the O-H stretching region (3800-3000 $\left.\mathrm{cm}^{-1}\right)$. The spectral intensity was normalized over the high wavenumber region. (E) $W_{C}$ dependence of the $\mathrm{OH}_{\text {Area }} / \mathrm{CH}_{\text {Area }}$ value. 

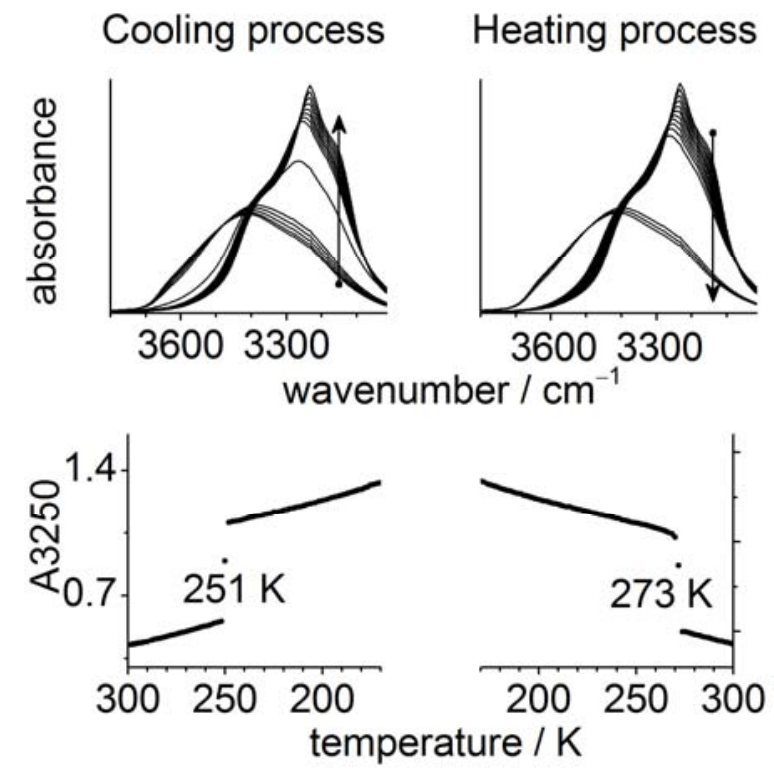

Figure 3. Temperature dependence of the mid-IR spectra (top) and A3250 value (bottom) of pure water. Left and right panels show the results of cooling and heating processes, respectively. 

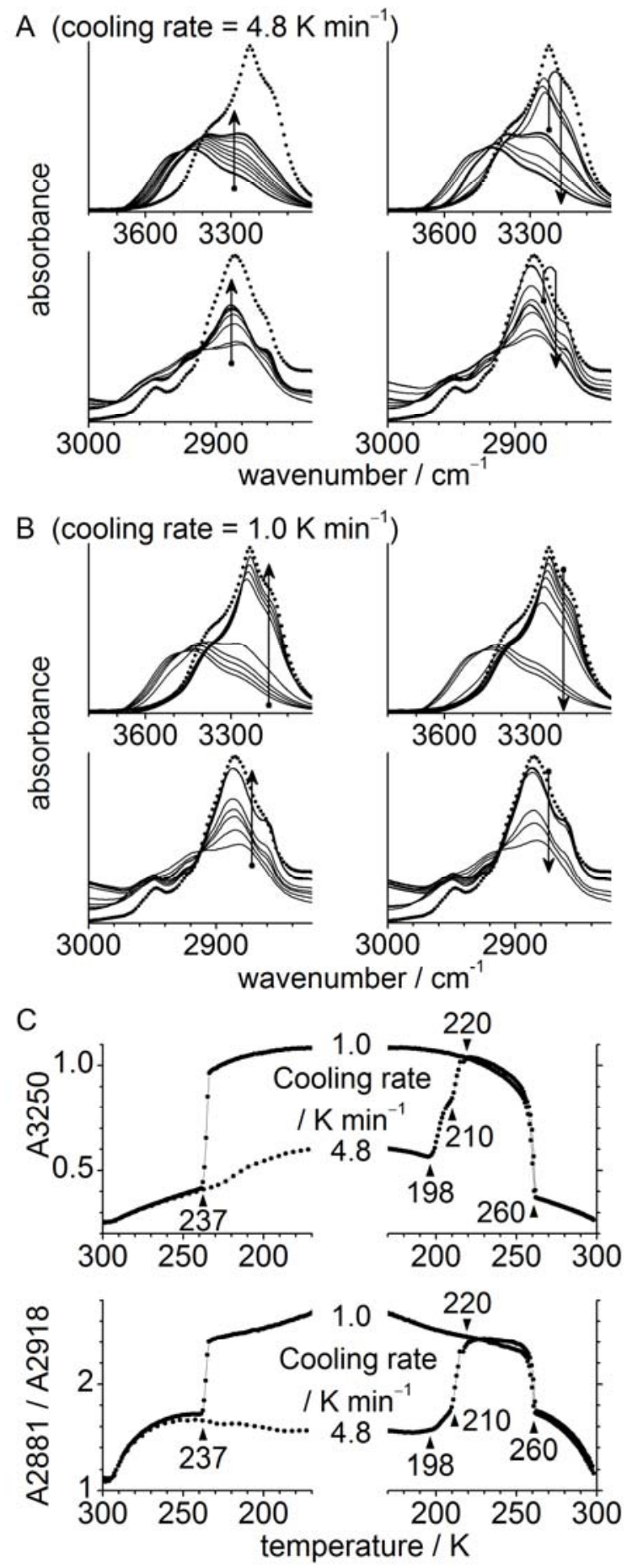

Figure 4. Temperature dependence of the mid-IR spectra of a PEG aqueous solution with a $W_{\mathrm{C}}$ of $38.6 \mathrm{wt} \%$. (A) Spectra with a cooling rate of $4.8 \mathrm{~K} \cdot \mathrm{min}^{-1}$. (B) Spectra with a cooling rate of $1.0 \mathrm{~K} \cdot \mathrm{min}^{-1}$. (C) Temperature dependence of $A 3250$ value (top) and that of A2881/A2918 value (bottom). The top and bottom panels of (A) and (B) are spectra of the $\mathrm{O}-\mathrm{H}$ stretching and $\mathrm{C}-\mathrm{H}$ stretching regions, respectively. The left and right parts of each panel are results of the cooling and heating processes, respectively. 
Cooling process Heating process
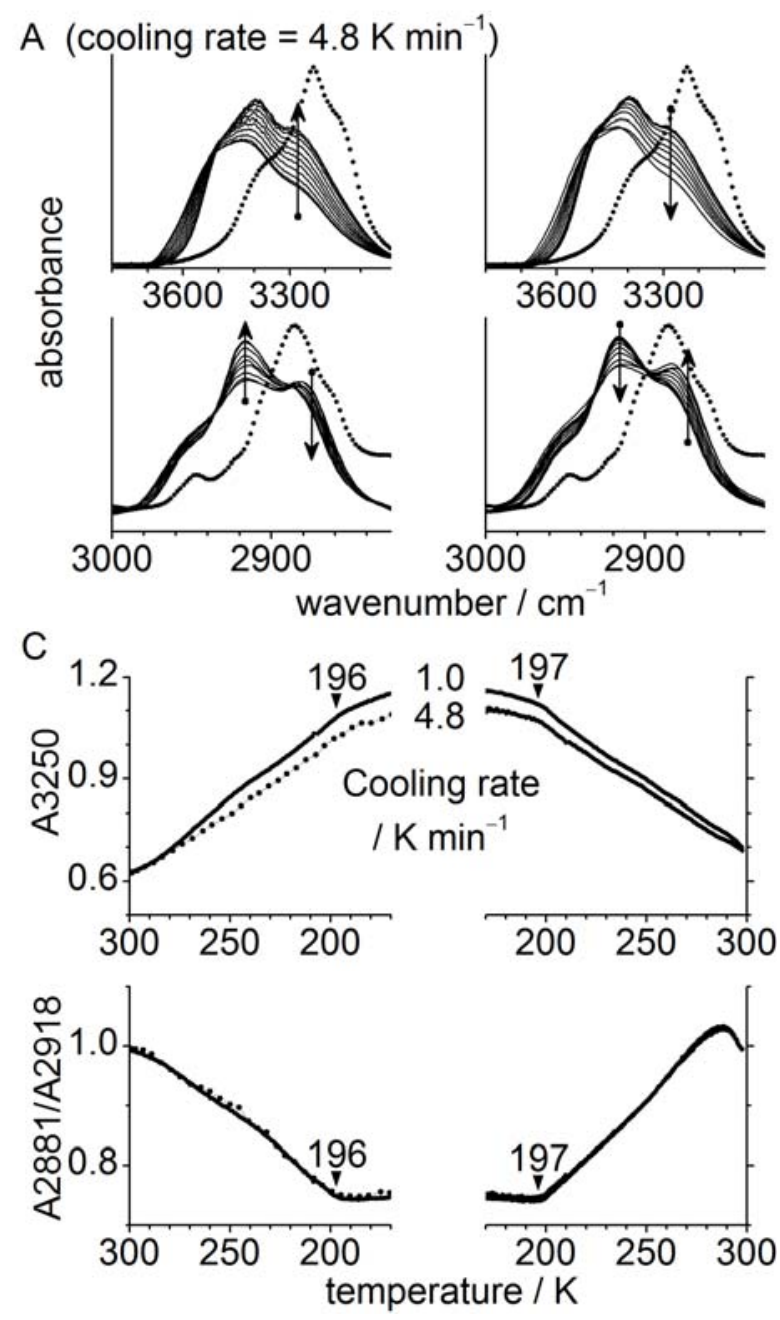

Figure 5. Temperature dependence of the mid-IR spectra of a PEG aqueous solution with a $W_{C}$ of $43.2 w t \%$. The details are the same as those in Figure 4 , though $(B)$ is omitted. 

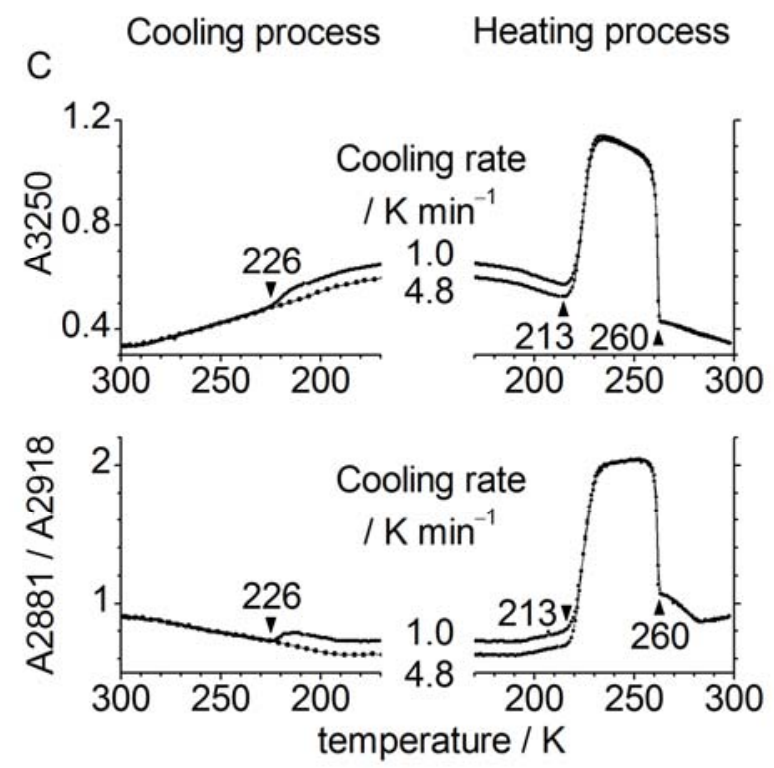

Figure 6. Temperature dependence of the mid-IR spectra of a PEG aqueous solution with a $W_{C}$ of $50.9 \mathrm{wt} \%$. The details are the same as those in Figure 4, though (A) and (B) are omitted. 
TOC

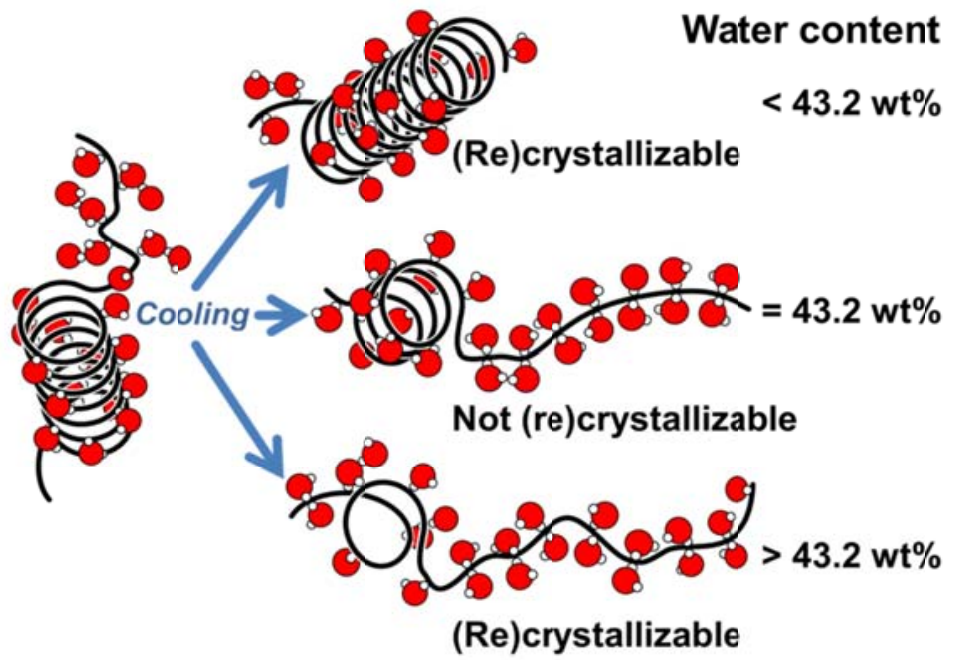

